\title{
Charge Generation and Electron-Trapping Dynamics in Hybrid Nanocrystal-Polymer Solar Cells
}

\author{
Frederik S. F. Morgenstern, ${ }^{\dagger}$ Marcus L. Böhm, ${ }^{\dagger}$ René J. P. Kist, Aditya Sadhanala, Simon Gélinas,
} Akshay Rao, and Neil C. Greenham*

Cavendish Laboratory, Department of Physics, University of Cambridge, J. J. Thomson Avenue, Cambridge, CB3 0HE, United Kingdom

Supporting Information

ABSTRACT: We investigate the charge-trapping dynamics in hybrid nanocrystalpolymer systems and their effect on performance in photovoltaic devices. Employing various steady-state spectroscopy techniques and ultrafast, three-pulse transient absorption methods, we identify the depth of electron trap states in the nanocrystal band gap and measure their population dynamics. Our findings show that photogenerated electrons are trapped at midgap states on the nanocrystal within hundreds of picoseconds. The trapping of the majority of charge carriers before charge extraction results in a lowering of the quasi-Fermi level of the electrons which limits the device open-circuit voltage, thereby underlining the significance of these processes in conjugated polymer/nanocrystal hybrid photovoltaics.

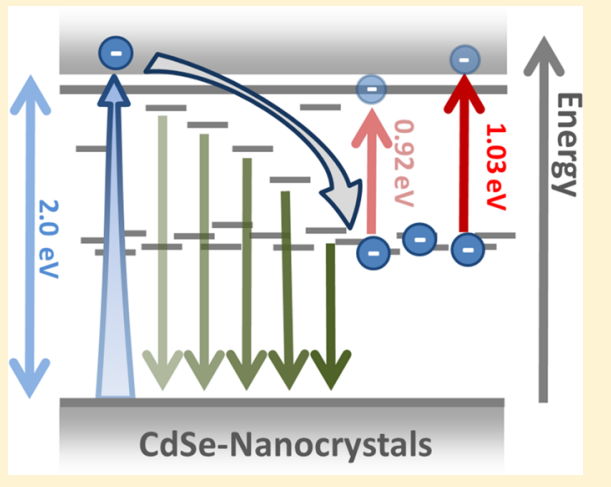

\section{INTRODUCTION}

Because of the prospect of cheap large-area production, organic photovoltaics have attracted great interest in the past decade. While an extensive library of high-performing donor polymers exists, the variety of electron acceptor materials is mainly restricted to fullerene derivatives. Alternative hybrid systems using inorganic semiconductor nanoparticles as the electron acceptor offer the advantages of additional absorption in the acceptor component, ${ }^{1}$ tunable energy levels through the quantum confinement effect, ${ }^{2,3}$ improved electron-hole separation efficiency because of increased screening of Coulombic interactions, ${ }^{4,5}$ and the potential to exploit carrier multiplication effects in the nanoparticles. ${ }^{6,7}$ However, the efficiency of hybrid bulk heterojunction photovoltaics still lags that of polymer:fullerene devices, largely because of operating voltages that are lower than expected from the intrinsic electronic energy levels of the materials involved. ${ }^{8,9}$

It is to be expected that electronic states associated with the nanoparticle surfaces will play an important role in hybrid device operation. ${ }^{5}$ Trapping of electrons in defect sites will, for a given carrier density, lower the quasi-Fermi level for electrons and thus limit the open-circuit voltage. ${ }^{10-12}$ Rapid trap assisted recombination may further reduce the open-circuit voltage by reducing the total steady-state carrier density. Several recent studies have investigated the processes of charge recombination and extraction in polymer/nanocrystal (NC) devices, ${ }^{4,5,8,13-15}$ but the operation of these devices is still less well understood than that of purely organic photovoltaics. This is largely due to the complexity of processes occurring at the nanoparticle surfaces and their interfaces with the organic component. ${ }^{16-18}$ Dynamical studies of electron trapping in various nanoparticle systems have been reported using transient absorption and time-resolved photoluminescence measurements, with trapping times ranging from picoseconds to nanoseconds. ${ }^{11,12,19,20}$

Surface states have been shown to be highly sensitive to the details of ligand coverage and local environment; ${ }^{5}$ hence, to improve device performance, it is important to understand the electron-trapping process directly in hybrid films. Here, we study the energetics and dynamics of electron trapping in hybrid nanocrystal-polymer films and solar cells using a combination of steady-state and transient spectroscopic techniques including three-pulse pump-push-probe measurements.

\section{RESULTS AND DISCUSSION}

Charge Dynamics in the Nano- and Microsecond Regime. In Figure 1a and b, we show the device architecture and solar cell characteristics of the model hybrid solar cell system used, consisting of the polymer donor poly[2-methoxy5-(3',7'-dimethyloctyloxy)-1,4-phenylenevinylene] (MDMOPPV) and $n$-butylamine ligated CdSe nanocrystals (diameter $6.5 \mathrm{~nm}$ ) as the electron acceptor. All the CdSe particles studied were synthesized in a single batch to avoid batch-to-batch variations. Detailed synthesis and device fabrication methods are provided in the Supporting Information S1. The opencircuit voltage of $0.7 \mathrm{~V}$ is significantly less than that seen in devices using the same polymer with $\operatorname{PCBM}([6,6]$-phenylC61-butyric acid methyl ester) as the electron acceptor, despite both acceptors having similar electron affinities (as estimated

Received: July 28, 2016

Published: August 1, 2016 
(a)

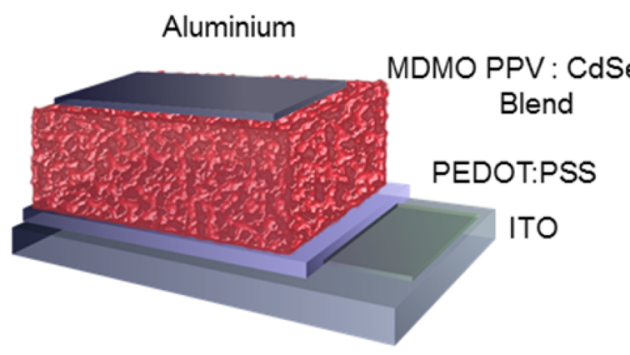

(c)

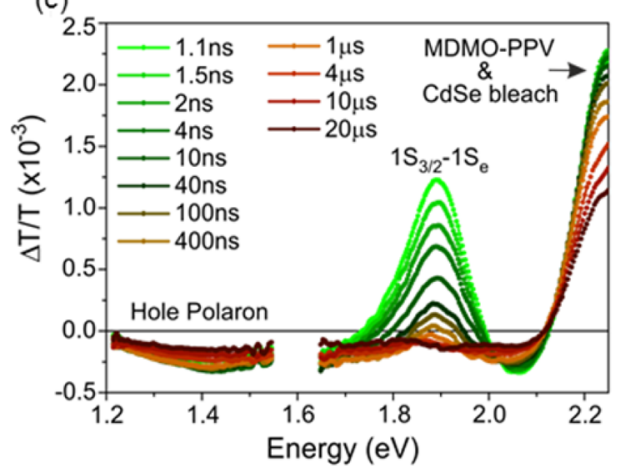

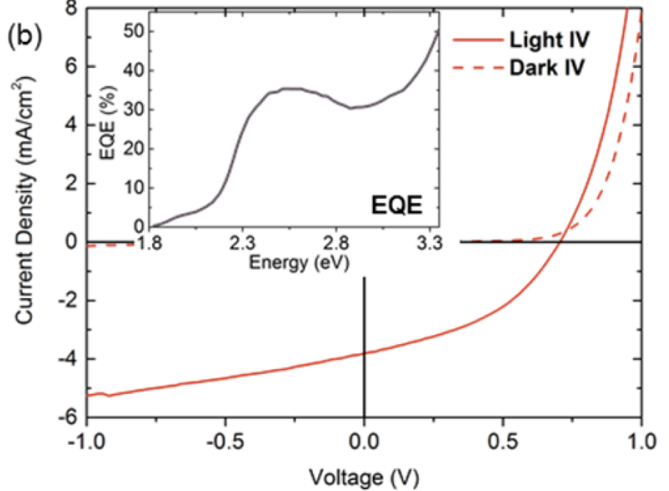

(d)

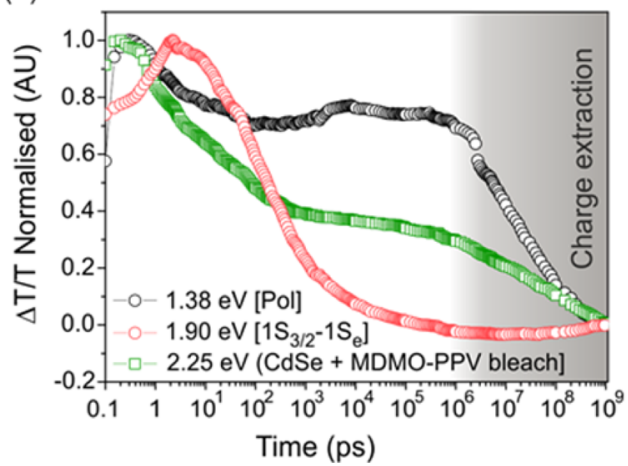

Figure 1. (a) Device architecture and (b) current-voltage characteristics of hybrid polymer-nanocrystal solar cells employing MDMO PPV as polymer donor and $n$-butylamine ligated CdSe NCs $(6.5 \mathrm{~nm}$ diameter) as electron acceptor species. The inset in $\mathrm{b}$ shows the corresponding external quantum efficiency. (c) Pump-probe transient absorption (TA) spectroscopy on the same devices excited at $2.3 \mathrm{eV}$ at a fluence of $4 \mu \mathrm{J} / \mathrm{cm}^{2}$. (d) TA kinetics of the polymer polarons (probed at $1.38 \mathrm{eV}$; black trace), the ground-state bleach $\left(\mathrm{GSB} ; 1 \mathrm{~S}_{3 / 2}-1 \mathrm{~S}_{\mathrm{e}}\right.$ transition) of the NCs (probed at $1.90 \mathrm{eV}$; red trace), and the combined bleach of NCs and polymer (probed at $2.25 \mathrm{eV}$; green trace).

from photoelectron spectroscopy and optical absorption measurements, see Supporting Information S2). This finding is consistent with a tail of trap states playing an important role in the nanoparticle-based devices.

To study the recombination and trapping processes in these hybrid solar cells, transient absorption (TA) experiments were performed on devices under short-circuit conditions. Figure 1c shows the TA spectrum following excitation at $2.3 \mathrm{eV}$ and measured in the nanosecond-to-microsecond time regime. The corresponding kinetic traces are displayed in Figure 1d which shows the combined bleach of NCs and polymer ${ }^{21}$ (2.25 eV; green), the $1 S_{3 / 2}-1 S_{e}$ transition (or ground-state bleach (GSB)) of CdSe NCs $\left(1.90 \mathrm{eV}\right.$; red), and the hole polaron of the polymer ${ }^{18}$ (1.38 eV; black). The NC GSB decays to ca. $20 \%$ of its original strength within the first $2 \mathrm{~ns}$ and decays fully within $1 \mu \mathrm{s}$. The polymer hole polaron, on the other hand, shows an initial decay to about $70 \%$ within 300 ps after which it reaches a long-lived plateau.

Transient photocurrent measurements conducted on the same hybrid cells showed charge extraction to occur on the microsecond time scale (see Supporting Information S4). We thus attribute the decay of the TA signals after $1 \mu$ s to the extraction of charge carriers. The initial TA decay kinetics at $2.25 \mathrm{eV}$ (which will initially be dominated by polymer GSB) are in good agreement with the quenching rates of polymer excitons observed in our previous studies. ${ }^{18}$ Furthermore, we ascribe the rise in bleach at $1.90 \mathrm{eV}$ between 0.4 and 2.2 ps to exciton transfer from the MDMO-PPV to the NCs. The bleach of the GSB of the CdSe NCs has been shown to arise from electrons occupying the lowest accessible $1 S$ state with only insignificant contributions of holes on the NCs. ${ }^{20-22}$ The rapid decay of this GSB could, in principle, be caused by rapid recombination of excitons or electrons on the NCs or by rapid charge extraction. However, the measured time scales for electron extraction (see Supporting Information S4) are much longer then the GSB decay time scales, and the devices display reasonably high quantum efficiencies when the NC component is excited (see inset of Figure 1b). We therefore rule out recombination and charge extraction as likely causes of the rapid GSB decay. Instead, we consider that the GSB decay is caused by photoexcited electrons making a transition from their initial bleaching states into states that are much less strongly coupled to the $1 S_{3 / 2}-1 S_{e}$ transition of the NCs. Deep trapping at surface states is a likely candidate for such a transition, and previous TA measurements on various nanoparticle systems have identified electron trapping as being responsible for GSB decay. ${ }^{11,12,19}$ However, most studies analyzing the electrontrapping dynamics have been performed on systems where luminescence measurements are possible. ${ }^{23-26}$

Trap Analysis via Steady-State Spectroscopy. To identify trap states residing within the band gap of the NC component, we monitor the intragap absorption profile of oleic acid ligated CdSe NCs using photothermal deflection spectroscopy (PDS). It is generally accepted that CdSe NCs passivated with their native ligands (i.e., oleic acid) exhibit fewer intragap states compared to particles which have been ligand-exchanged to $n$-butylamine, since the ligand-exchange process is likely to create additional surface trap states. ${ }^{5}$ Indeed, comparing the absorbance profile of $n$-butylamine- and oleic acid ligated CdSe NCs, we find a sharper absorbance onset in the latter NC system (see Supporting Information S5). 
Furthermore, because of the higher achievable solution concentration of OA-ligated NCs (ca. $200 \mathrm{mg} / \mathrm{mL}$ ), we could produce thicker films, which enhances the measurement accuracy of PDS at low trap state densities. We therefore consider the abundance of trap states in oleic acid ligated NCs as a lower limit for the analogous $n$-butylamine passivated NCs. The absorption profile shown in Figure 2a reveals a long tail of
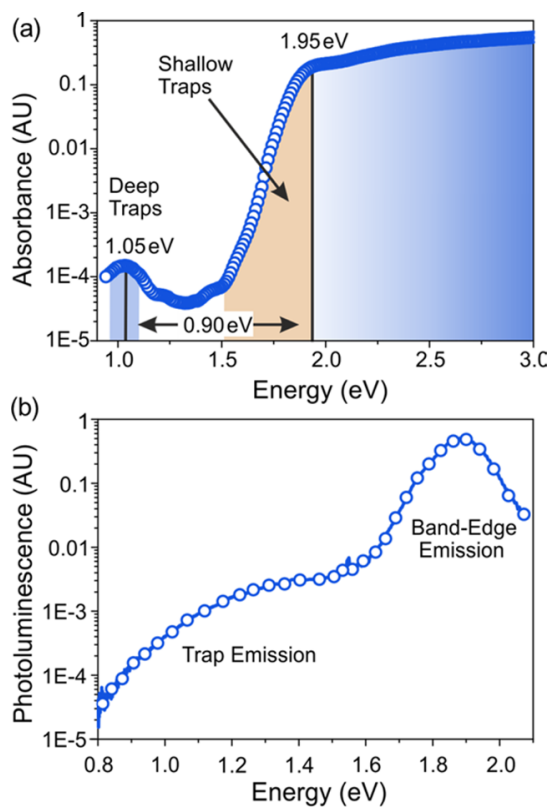

Figure 2. (a) Photothermal deflection spectroscopy of oleic acid ligated CdSe NCs in film. A comparison between the spectra of oleic acid and $n$-butylamine-ligated particles is provided in the Supporting Information S5. (b) Photoluminescence of the same NC films.

shallow trap states extending from $1.95 \mathrm{eV}$ down to $1.50 \mathrm{eV}$. Interestingly, we observe a rise in signal intensity at ca. $1 \mathrm{eV}$ which we assign to a direct absorption process into midgap states. This picture is in agreement with steady-state photoluminescence measurements where we find spectrally broad emission in the near-infrared $(>0.8 \mathrm{eV})$ which is consistent with emission from sub-band-gap trap states ${ }^{20}$ (see Figure $2 b$ ). Having identified the presence of both shallow and midgap trap states, we will next study the kinetics of their filling with photogenerated charge carriers.

Trap Analysis via Transient Spectroscopy. Using transient pump-push-probe experiments allows us to directly monitor the kinetics of electron trapping in the CdSe NC devices. In conventional TA, the sample is pumped at time $t=0$ and is probed at time $t$. Here, we add an additional push at time $t_{\text {push }}$ between pump and probe pulses. Performing control experiments without the initial pump pulse allows us to account for parasitic signals such as two-photon absorption (see Supporting Information S6 for more details) and thus to monitor changes in transmission caused by interaction of the push signal with photoexcited carrier species.

A tunable push pulse of excitation energy ranging between 1.03 and $0.89 \mathrm{eV}$ was applied at $t_{\text {push }}=1.3$ ns. Figure 3 shows the kinetics of the push-induced change in transmission $\Delta(\Delta T / T)$ probed at the $1 S_{3 / 2}-1 S_{\mathrm{e}}$ transition $(1.90 \mathrm{eV})$ for a series of push energies. To account for variations in flux at the different push energies, the push-induced $\Delta(\Delta T / T)$ signals were scaled to the photon density of the $1.03 \mathrm{eV}$ push pulse (see Supporting Information S6).

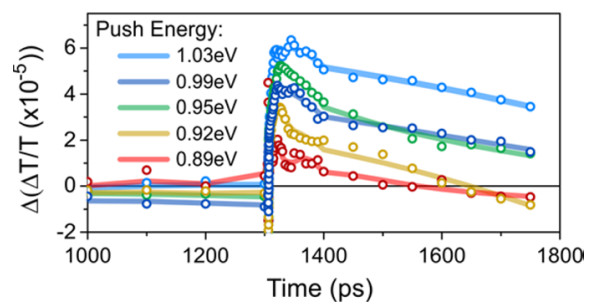

Figure 3. Transient absorption measurement using pump-pushprobe techniques. Devices were excited using a $2.25 \mathrm{eV}$ pump pulse and were then pushed after $1.3 \mathrm{~ns}$ using an IR push pulse of energy between 0.89 and $1.03 \mathrm{eV}$. The kinetics shown here were rescaled to account for differences in photon densities between the different push pulses.

When applying a push pulse of $1.03 \mathrm{eV}$, we observe a strong positive signal indicating an additional bleach signal with a longlived component. The spectral shape of the push-induced $\Delta(\Delta T / T)$ signal resembles the GSB of the unperturbed system (see Figure 1c). For push pulses of $0.99 \mathrm{eV}$, we find a similar behavior albeit with a weaker response. When pushing with $0.95 \mathrm{eV}$, the induced GSB is found to decay even more quickly, and at $0.92 \mathrm{eV}$, it fully decays within $300 \mathrm{ps}$ after the push pulse. When pushing at lower energies $(0.89 \mathrm{eV})$, we observe only very weak and short-lived responses to the push pulse.

We attribute this positive bleach to the recovery of the $\mathrm{NC}$ GSB induced by the detrapping of electrons (we consider the contribution of the hole dynamics to be insignificant because of the difference in effective mass $\left.{ }^{18,21}\right)$. Here, the push pulse has enough energy to promote an electron from a midgap state to the conduction band, thus bleaching the $1 S_{3 / 2}-1 S_{\mathrm{e}}$ transition of the NCs. Electrons which experience push pulse energies of $0.92 \mathrm{eV}$ have just enough energy to be detrapped but do not fully escape the environment of the trap site, leading to a shortlived bleach signal. Applying higher push energies promotes the deeply trapped electrons into a more delocalized state within the conduction band, leading to a longer-lived GSB recovery. The energetic onset of $0.92 \mathrm{eV}$ at which the push pulse is able to induce a long-lived bleach is in good agreement with the midgap states measured by PDS and photoluminescence (PL) spectroscopy (see Figure 2).

To obtain a more detailed picture of the trapping dynamics, we monitored the push response as a function of pump-push delay $\left(t_{\text {push }}\right)$ at a constant push energy of $1.03 \mathrm{eV}$. Figure $4 \mathrm{a}$ shows the push-induced transient absorption spectra measured 20 ps after the push pulse for $t_{\text {push }}$ values between 9 and 1300 ps. For short pump-push delays $(<100 \mathrm{ps})$, the spectrum is derivative-like, but at longer delays, the spectrum changes to approach the GSB spectrum observed in Figure 1c. Timeresolved spectra for each push delay can be found in the Supporting Information S5. The first-derivative-like feature found for short pump-push delays, which decays very rapidly after the push pulse (Figure $4 \mathrm{~b}$ ), can arise because of a pushinduced change in polarization resulting in a Stark shift or through band gap renormalization effects. ${ }^{27,28}$ The lack of a push-induced GSB recovery suggests that detrapping of charges plays a less important role when the push arrives less than 100 ps after the pump, since most electrons have not had time to be trapped. At longer pump-push delays, the signal is consistent with a model in which the pump produces carriers which are trapped into nonbleaching states on time scales of hundreds of picoseconds (consistent with the TA data in Figure 1d). These carriers can then be re-excited to bleaching states by the push 

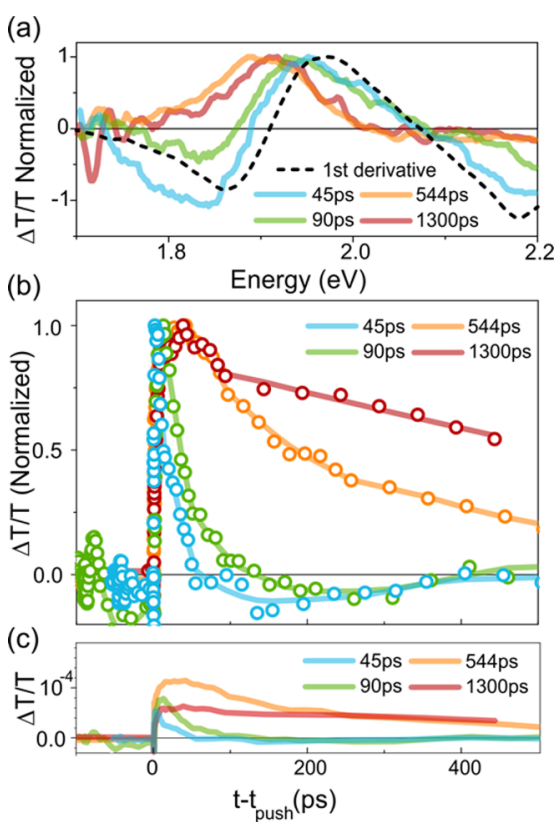

Figure 4. (a) The normalized TA spectra for different pump-push delays measured 20 ps after the push pulse. The dotted line depicts the first derivative of the ground-state bleach. (b) Normalized and (c) non-normalized transient pump-push-probe kinetics measured at $1.90 \mathrm{eV}$ (GSB of the NCs) at pump and push energies of 2.30 and $1.03 \mathrm{eV}$, respectively.

pulse from where they are again eventually trapped on time scales of hundreds of picoseconds. The trapping dynamics are consistent with a carrier diffusion mediated trapping process as suggested by Bakulin et al. ${ }^{19}$

The strength and dynamics of the push-induced signal can provide additional detail about different populations of trap states that may be present. For example, Figure $4 \mathrm{c}$ shows that the GSB recovery at $t_{\text {push }}=1300$ ps is smaller by almost a factor of 2 than the signal measured at $t_{\text {push }}=544$ ps and decays more slowly. The change in signal strength could simply be associated with recombination of trapped charges, although it is unlikely that this is the predominant factor since such rapid recombination would be incompatible with the photocurrents measured. It is more likely that the observed behavior is associated with time-dependent filling of traps of different depths, with deeper traps having a smaller optical cross section to the push pulse. Burda et al. have shown that shallow trap states within the NC band gap are populated earlier than their deeper counterparts, ${ }^{20}$ which is consistent with this picture. For the $1.03 \mathrm{eV}$ push pulse energy used here, electrons populating both shallow and deep trapped sites would be promoted into the conduction band, albeit to different positions within the density of states. It is unclear as of yet why pushing electrons out of shallow traps would lead to a shorter-lived GSB recovery than when pushing electrons out of midgap states, particularly when higher pump energies are found to produce longer GSB recovery times. However, different CdSe NCs can have different trap states, ${ }^{29-31}$ and so to form a full picture, it will be necessary to consider different populations of NCs interacting differently with push and probe, including the possibility of transfer of mobile electrons between NCs.

On the basis of the measurements presented, we propose a model for hybrid device operation where the majority of photogenerated electrons localize on midgap traps prior to their extraction.
As a consequence, the large quantity of populated deep traps lowers the quasi-Fermi level of the electrons in the CdSe NCs toward the middle of the NC band gap energetically located at about $-4.7 \mathrm{eV}$ (see Figure 5). ${ }^{32}$ A quasi-Fermi level of $0.9 \mathrm{eV}$

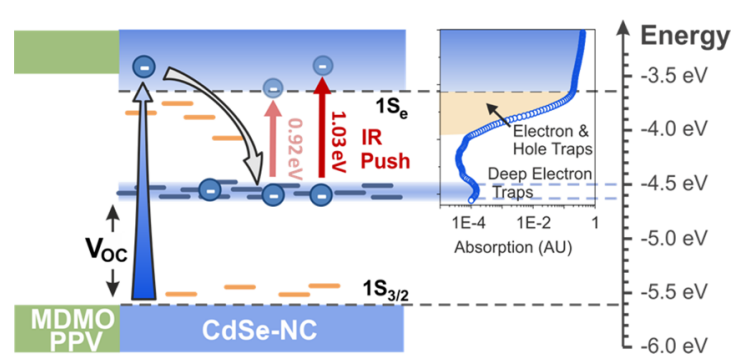

Figure 5. Energy diagram of the trap levels in CdSe NCs and proposed electron-trapping mechanism for photogenerated charge carriers which results in a limitation of the maximum achievable $V_{\mathrm{OC}}$.

below the conduction band of the CdSe NC can also explain the relatively low $V_{\mathrm{OC}}$ of ca. $0.7 \mathrm{~V}$ (see Figure $1 \mathrm{~b}$ ), indicating that deep traps in the NCs are a limiting factor in the device performance. To rule out that this low $V_{\mathrm{OC}}$ is caused by Fermi level pinning at the electron-extracting electrode, we prepared a range of MDMO-PPV:CdSe NC devices where we move the electron collection electrode energetically closer to the vacuum level by using lower-workfunction materials (see Supporting Information S7). As we do not observe an improved higher $V_{\mathrm{OC}}$, we argue that Fermi level pinning at the aluminum contact does not play a major role in these devices. This presents further evidence that low $V_{\mathrm{OC}}$ 's in these NC-containing hybrid devices are a consequence of electron trap states located in the middle of the band gap (see Figure 5).

\section{CONCLUSIONS}

We have used a range of steady-state and transient spectroscopy techniques to monitor the population of trap states in CdSe NCs in operational hybrid photovoltaic devices. We find that the $1 S_{3 / 2}-1 S_{e}$ transition bleach of CdSe NCs decays fully before charge extraction occurs, and we assign electron trapping as responsible for this quench in GSB. These electron traps were found to be located ca. $0.9 \mathrm{eV}$ below the NC conduction band and to be populated on time scales of hundreds of picoseconds. Trapping of the majority of electrons before charge extraction in the CdSe NCs leads to a lowering of the quasi-Fermi level to ca. $0.9 \mathrm{eV}$, which is consistent with the low $V_{\mathrm{OC}}$ observed in these devices. This suggests that future improvements in NC trap passivation could lead to significant enhancements in device $V_{\mathrm{OC}}$ and, therefore, in solar cell performance, enabling hybrid polymer:NC solar cells to surpass their polymer-fullerene counterparts.

\section{ASSOCIATED CONTENT}

\section{S Supporting Information}

The Supporting Information is available free of charge on the ACS Publications website at DOI: 10.1021/acs.jpcc.6b07591.

Experimental methods, ultraviolet photoelectron spectroscopy (UPS), supplemental current voltage characteristics, and transient photocurrent measurements (TPC) as well as detailed description and raw spectra of the pumppush-probe transient absorption experiments (PDF) 


\section{AUTHOR INFORMATION}

\section{Corresponding Author}

*E-mail: ncg11@cam.ac.uk; phone: +44 (0)1223 766301.

\section{Author Contributions}

${ }^{\dagger}$ F.S.F.M. and M.L.B. contributed equally to this work. The manuscript was written through contributions of all authors. All authors have given approval to the final version of the manuscript.

Notes

The authors declare no competing financial interest.

\section{ACKNOWLEDGMENTS}

This work was supported by the Engineering and Physical Sciences Research Council (grant numbers EP/M005143/1, EP/G060738/1, EP/G037221/1). F.S.F.M. gratefully acknowledges the Worshipful Company of Armourers and Brasiers for support with the Gauntlet Trust award, and M.L.B thanks the German National Academic Foundation (Studienstiftung) for funding. The data underlying this paper are available at http://dx.doi.org/10.17863/CAM.915.

\section{REFERENCES}

(1) Greenham, N. C. Hybrid Polymer/Nanocrystal Photovoltaic Devices. In Organic Photovoltaics; Brabec, C., Dyakonov, V., Scherf, U., Eds.; Wiley-VCH: Weinheim, Germany, 2008.

(2) Alivisatos, A. P. Semiconductor Clusters, Nanocrystals, and Quantum Dots. Science 1996, 271, 933-937.

(3) Böhm, M. L.; Jellicoe, T. C.; Rivett, J.; Sadhanala, A.; Davis, N. J. L. K.; Morgenstern, F. S. F.; Goedel, K. C.; Govindasamy, J.; Benson, C. G. M.; Greenham, N. C.; et al. Size and Energy Level Tuning of Quantum Dot Solids via a Hybrid Ligand Complex. J. Phys. Chem. Lett. 2015, 6, 3510-3514.

(4) Maclachlan, A. J.; Rath, T.; Cappel, U. B.; Dowland, S. A.; Amenitsch, H.; Knall, A.; Buchmaier, C.; Trimmel, G.; Nelson, J.; Haque, S. A. Polymer/Nanocrystal Hybrid Solar Cells: Influence of Molecular Precursor Design on Film Nanomorphology, Charge Generation and Device Performance. Adv. Funct. Mater. 2015, 25, 409-420.

(5) Böhm, M. L.; Kist, R. J. P.; Morgenstern, F. S. F.; Ehrler, B.; Zarra, S.; Kumar, A.; Vaynzof, Y.; Greenham, N. C. The Influence of Nanocrystal Aggregates on Photovoltaic Performance in NanocrystalPolymer Bulk Heterojunction Solar Cells. Adv. Energy Mater. 2014, 4, 1400139.

(6) Davis, N. J. L. K.; Böhm, M. L.; Tabachnyk, M.; WisniveskyRocca-Rivarola, F.; Jellicoe, T. C.; Ducati, C.; Ehrler, B.; Greenham, N. C. Multiple-Exciton Generation in Lead Selenide Nanorod Solar Cells with External Quantum Efficiencies Exceeding 120\%. Nat. Commun. 2015, 6, 8259 .

(7) Böhm, M. L.; Jellicoe, T. C.; Tabachnyk, M.; Davis, N. J. L. K.; Wisnivesky Rocca Rivarola, F.; Ducati, C.; Ehrler, B.; Bakulin, A. A.; Greenham, N. C. Lead Telluride Quantum Dot Solar Cells Displaying External Quantum Efficiencies Exceeding 120\%. Nano Lett. 2015, 15, 7987-7993.

(8) Greaney, M. J.; Das, S.; Webber, D. H.; Bradforth, S. E.; Brutchey, R. L. Improving Open Circuit Potential in Hybrid P3HT:CdSe Bulk Heterojunction Solar Cells via Colloidal TertButylthiol Ligand Exchange. ACS Nano 2012, 6, 4222-4230.

(9) Lek, J. Y.; Lam, Y. M.; Niziol, J.; Marzec, M. Understanding Polycarbazole-Based polymer:CdSe Hybrid Solar Cells. Nanotechnology 2012, 23, 315401.

(10) Gao, F.; Li, Z.; Wang, J.; Rao, A.; Howard, I. a; Abrusci, A.; Massip, S.; McNeill, C. R.; Greenham, N. C. Trap-Induced Losses in Hybrid Photovoltaics. ACS Nano 2014, 8, 3213-3221.

(11) Boehme, S. C.; Azpiroz, J. M.; Aulin, Y.; Grozema, F. C.; Vanmaekelbergh, D.; Siebbeles, L. D. A.; Infante, I.; Houtepen, A. J.
Density of Trap States and Auger-Mediated Electron Trapping in CdTe Quantum-Dot Solids. Nano Lett. 2015, 15, 3056-3066.

(12) Boehme, S. C.; Walvis, T. A.; Infante, I.; Grozema, F. C.; Vanmaekelbergh, D.; Siebbeles, L. D. a; Houtepen, A. J. Electrochemical Control over Photoinduced Electron Transfer and Trapping in CdSe-CdTe Quantum-Dot Solids. ACS Nano 2014, 8, 7067-7077.

(13) Couderc, E.; Greaney, M. J.; Brutchey, R. L.; Bradforth, S. E. Direct Spectroscopic Evidence of Ultrafast Electron Transfer from a Low Band Gap Polymer to CdSe Quantum Dots. J. Am. Chem. Soc. 2013, 135, 18418.

(14) Greaney, M. J.; Brutchey, R. L. Ligand Engineering in Hybrid Polymer:nanocrystal Solar Cells. Mater. Today 2015, 18, 31-38.

(15) Dowland, S. A.; Reynolds, L. X.; MacLachlan, A.; Cappel, U. B.; Haque, S. A. Photoinduced Electron and Hole Transfer in CdS:P3HT Nanocomposite Films: Effect of Nanomorphology on Charge Separation Yield and Solar Cell Performance. J. Mater. Chem. A 2013, 1, 13896

(16) Strein, E.; Colbert, A.; Subramaniyan, S.; Nagaoka, H.; Schlenker, C. W.; Janke, E.; Jenekhe, S. A.; Ginger, D. S. Charge Generation and Energy Transfer in Hybrid Polymer/infrared Quantum Dot Solar Cells. Energy Environ. Sci. 2013, 6, 769.

(17) Strein, E.; DeQuilettes, D.; Hsieh, S. T.; Colbert, A. E.; Ginger, D. S. Hot Hole Transfer Increases Polaron Yields in Hybrid Conjugated-Polymer/PbS Blends. J. Phys. Chem. Lett. 2014, 5, 208211.

(18) Morgenstern, F. S. F.; Rao, A.; Böhm, M. L.; Kist, J. P.; Vaynzof, Y.; Greenham, N. C. Ultrafast Charge- and Energy-Transfer Dynamics in Conjugated Polymer: Cadmium Selenide Nanocrystal Blends. ACS Nano 2014, 8, 1647-1654.

(19) Bakulin, A. A.; Neutzner, S.; Bakker, H. J.; Ottaviani, L.; Barakel, D.; Chen, Z. Charge Trapping Dynamics in PbS Colloidal Quantum Dot Photovoltaic Devices. ACS Nano 2013, 7, 8771-8779.

(20) Burda, C.; Link, S.; Mohamed, M.; El-Sayed, M. The Relaxation Pathways of CdSe Nanoparticles Monitored with Femtosecond TimeResolution from the Visible to the IR: Assignment of the Transient Features by Carrier Quenching. J. Phys. Chem. B 2001, 105, 1228612292.

(21) Klimov, V.; McBranch, D. Electron and Hole Relaxation Pathways in Semiconductor Quantum Dots. Phys. Rev. B: Condens. Matter Mater. Phys. 1999, 60, 13740-13749.

(22) Klimov, V. I. Spectral and Dynamical Properties of Multiexcitons in Semiconductor Nanocrystals. Annu. Rev. Phys. Chem. 2007, $58,635-673$

(23) Underwood, D. F.; Kippeny, T.; Rosenthal, S. J. Ultrafast Carrier Dynamics in CdSe Nanocrystals Determined by Femtosecond Fluorescence Upconversion Spectroscopy. J. Phys. Chem. B 2001, $105,436-443$.

(24) Saari, J. I.; Dias, E. A.; Reifsnyder, D.; Krause, M. M.; Walsh, B. R.; Murray, C. B.; Kambhampati, P. Ultrafast Electron Trapping at the Surface of Semiconductor Nanocrystals: Excitonic and Biexcitonic Processes. J. Phys. Chem. B 2013, 117, 4412-4421.

(25) Mooney, J.; Krause, M.; Saari, J.; Kambhampati, P. Challenge to the Deep-Trap Model of the Surface in Semiconductor Nanocrystals. Phys. Rev. B: Condens. Matter Mater. Phys. 2013, 87, 081201.

(26) Jones, M.; Lo, S. S.; Scholes, G. D. Quantitative Modeling of the Role of Surface Traps in CdSe/CdS/ZnS Nanocrystal Photoluminescence Decay Dynamics. Proc. Natl. Acad. Sci. U. S. A. 2009, 106, 3011-3016.

(27) Gélinas, S.; Rao, A.; Kumar, A.; Smith, S.; Chin, A. Ultrafast Long-Range Charge Separation in Organic Semiconductor Photovoltaic Diodes. Science 2014, 343, 512-517.

(28) Price, M. B.; Butkus, J.; Jellicoe, T. C.; Sadhanala, A.; Briane, A.; Halpert, J. E.; Broch, K.; Hodgkiss, J. M.; Friend, R. H.; Deschler, F. Hot Carrier Cooling and Photoinduced Refractive Index Changes in Organic-Inorganic Lead Halide Perovskites. Nat. Commun. 2015, 6, 8420.

(29) Ebenstein, Y.; Mokari, T.; Banin, U. Fluorescence Quantum Yield of CdSe/ZnS Nanocrystals Investigated by Correlated Atomic- 
Force and Single-Particle Fluorescence Microscopy. Appl. Phys. Lett. 2002, 80, 4033-4035.

(30) Pons, T.; Medintz, I. L.; Farrell, D.; Wang, X.; Grimes, A. F.; English, D. S.; Berti, L.; Mattoussi, H. Single-Molecule Colocalization Studies Shed Light on the Idea of Fully Emitting versus Dark Single Quantum Dots. Small 2011, 7, 2101-2108.

(31) Jellicoe, T. C.; Richter, J. M.; Glass, H. F. J.; Tabachnyk, M.; Brady, R.; Dutton, S. E.; Rao, A.; Friend, R. H.; Credgington, D.; Greenham, N. C.; et al. Synthesis and Optical Properties of Lead-Free Cesium Tin Halide Perovskite Nanocrystals. J. Am. Chem. Soc. 2016, 138, 2941-2944.

(32) Li, Z.; Wang, W.; Greenham, N. C.; McNeill, C. R. Influence of Nanoparticle Shape on Charge Transport and Recombination in Polymer/nanocrystal Solar Cells. Phys. Chem. Chem. Phys. 2014, 16, 25684-25693. 